EPiC Series in Engineering
Volume 3, 2018, Pages 1289-1297
HIC 2018. 13th International
Conference on Hydroinformatics

\title{
Influence of climate change on the optimization of water supply systems
}

\author{
Mario Maiolo $^{1^{*}}$, Giuseppe Mendicino ${ }^{1 \dagger}$, Daniela Pantusa ${ }^{2 \ddagger}$, Alfonso \\ Senatore ${ }^{1 \S}$ and Joaquim Sousa ${ }^{3,4^{* *}}$ \\ ${ }^{1}$ University of Calabria, Department of Environmental and Chemical Engineering, Rende, Italy \\ ${ }^{2}$ University of Salento, Innovation Engineering Department, Lecce, Italy \\ ${ }^{3}$ Polytechnic Institute of Coimbra, Department of Civil Engineering, Coimbra, Portugal \\ ${ }^{4}$ INESC C - Institute for Systems Engineering and Computers, FCTUC, Coimbra, Portugal \\ mario.maiolo@unical.it, giuseppe.mendicino@unical.it, \\ alfonso.senatore@unical.it, daniela.pantusa@unisalento.it, \\ jjoseng@isec.pt
}

\begin{abstract}
In the last decades, climate change is affecting several aspects of human and natural systems worldwide. Concerning water resources, the main impacts are related to the combined effect of temperature increase and changes in availability and distribution of precipitation, which affects both quantity and quality. The Mediterranean is potentially very sensitive to climate change. In Calabria (Southern Italy) the projected reduction suggests a particular care in matching water resource availability and needs. In this paper, the province of Crotone in Calabria was analyzed as a study case. This area is characterized by a sufficient availability of resources as a whole when compared with the needs of the users, but with an unbalanced distribution through its networks. This condition requires the identification of a resource allocation optimization solution. Using a least-cost optimization model, water resource optimization solutions were identified and compared starting from a review of the existing water supply systems, taking into account both current water availability and possible future availability due to climate change.
\end{abstract}

\footnotetext{
* Performed the optimization analysis,

${ }^{\dagger}$ Performed the hydrological analysis for both the current period and the future scenario

$\star$ Performed the optimization analysis

$\S$ Performed the hydrological analysis for both the current period and the future scenario

*** Performed the optimization analysis
} 


\section{Introduction}

The amount of water available for withdrawal is a function of runoff, groundwater recharge, aquifer conditions, water quality and water supply infrastructure. Safe access to drinking water depends more on the level of water supply infrastructure than on the quantity of available runoff. However, the goal of improved safe access to drinking water will be harder to achieve in regions where runoff and/or groundwater recharge decreases as a result of climate change. In addition, climate change leads to additional costs for the water supply sector, which might hamper the extension of water supply services to more people. This leads, in turn, to higher socio-economic impacts and follow-up costs, especially in areas where the prevalence of water stress has also increased as a result of climate change (Bates, Kundzewicz, Wu, \& Palutikof, 2008). In this context, the concept of correct management of water supply systems includes the ability to organize a distribution system capable to ensure proper supply of the resource, even in relation to changes in demand or water availability. Therefore, appropriate optimization strategies are necessary, and the proper allocation of available resources is becoming increasingly important in order to achieve an optimal management of such type of systems. For these systems the goal of achieving the lowest water usage rate is of interest not only in relation to operating costs and economic management optimization, but also in relation to financial optimization of investment plans. In fact, the optimization of new pipelines has a positive impact on water tariffs. With the aim of proper management of investment plans, there is a need for fast and accurate methodological forecasting tools for water supply systems optimization which are capable of accounting for possible future scenarios of reduction in water availability due to the effects, e.g., of climate change or evolution of demand. In this paper an optimization solution accounting for the expected reduced availability of water resources in the context of climate change was created and compared with the optimization solution for current water availability.

\section{Material and Methods}

Climate change impact has been assessed by means of a simulation provided by the COSMO-Climate Limited-area Modeling (CCLM) (Rockel \& Geyer, 2008) Regional Climate Model (RCM) using the Representative Concentration Pathway (RCP) 4.5 (Moss, et al., 2010) in the context of the EUROCORDEX initiative (www. euro-cordex.net; a detailed description of the experiment can be found in (Jacob, et al., 2014). The CCLM model performed dynamic downscaling of the MPI-ESM-LR General Circulation Model (GCM) and was applied at a resolution of $0.11^{\circ}$ (approximately $12.5 \mathrm{~km}$ ) both during the control period (1971-2005) and the future scenario (2016-2050). Further downscaling at an adequate resolution for the hydrological analysis needed in this study was performed with a distributed water balance model (Mendicino, Senatore, \& Versace, A Groundwater Resource Index (GRI) for drought monitoring and forecasting in a mediterranean climate, 2008). The model simulated soil moisture and groundwater content dynamics, snow accumulation and melting, evapotranspiration, baseflow and subsurface/surface runoff on a 5-km resolution regular grid with a monthly time step, using temperature and precipitation provided by the RCM as climatic drivers. In addition, vegetation and soil properties were also needed as static input for the model, but they were considered constant over time both in the control period and future scenarios. The reliability of the water balance model has been widely tested on the entire regional territory of Calabria ( (Mendicino, Senatore, \& Versace, A Groundwater Resource Index (GRI) for drought monitoring and forecasting in a mediterranean climate, 2008), 
(Mendicino, Senatore, \& Versace, Water resources management in agriculture under drought and water shortage conditions: A case study in southern Italy, 2008) (Cervarolo, Mendicino , \& Senatore, 2012)). The model, which assumed five years of acceleration in both the control period and in the future scenario, was able to assess changes in water resources availability for the climate change scenario and to proceed to the water scheme optimization.

For the optimization model, the present paper resumes and revises a least-cost optimization model proposed in ( (Maiolo \& Pantusa, 2016), (Carini , Maiolo, Pantusa, Chiaravalloti, \& Capano, 2017)) aimed at identifying the proper allocation of drinking water resources. In particular, the optimization model analyses the water resources available in a territory in relation to the demand of the users, in order to determine the possible transfer of water resources in the territory by different schemes. The rationalization of water resources can be obtained through a review of the existing drinking water systems and the identification of new pipelines to be built.

The model returns as output an ideal water supply system which is able to achieve optimal allocation in terms of the minimum overall cost for the completion of the supply system.

The optimization model was developed in MATLAB which allows an accurate and expeditious computational evaluation of the optimal solutions.

The model is defined as follows:

- given $m$ source nodes (springs, wells, intakes), each one characterized by a water availability (average daily volume) labeled as $a_{i}$, where $\mathrm{i}=1,2, \ldots, \mathrm{m}$;

- and fixed $n$ destination nodes (users), each one characterized by a user demand labeled as $b_{j}$, where $\mathrm{j}=1,2, \ldots, \mathrm{n}$;

- with the average day volume $V_{i j}\left(\mathrm{~m}^{3}\right)$ transferred from source node $i$ to destination node $j$;

- and the cost $C_{i j}$ of the transferring of $V_{i j}$

Then, the optimal allocation configuration is the one that minimizes the total cost of the whole water supply system:

$$
C=\sum_{j=1}^{n} \sum_{i=1}^{m} C_{i j}
$$

With reference to the research activity and optimization model described in ( (Maiolo \& Pantusa, 2016), (Carini , Maiolo, Pantusa, Chiaravalloti, \& Capano, 2017), (Maiolo, Mendino, Pantusa , \& Senatore, 2017)) this work proposes an improvement of the cost modeling previously proposed in ( (Maiolo \& Pantusa, 2016), (Carini , Maiolo, Pantusa, Chiaravalloti, \& Capano, 2017)) through: 1) Inclusion of existing pipes with new ones in parallel if necessary; 2) Inclusion of pumping stations (existing and new ones when needed); 3) Inclusion of operation costs for the pumping stations (existing and new ones when needed); 4) Design for adequate peak flows and consideration of operating costs (energy costs) based on average flows.

The following assumptions are used for the cost modeling:

Flows: gravity flow pipe $-Q=\frac{V \cdot P_{f}}{3600 N H_{\max , g}}$; pumping station $-Q=\frac{V \cdot P_{f}}{3600 N H_{\max , p}}$

Head loss formula: $J=\frac{\beta \cdot Q^{\delta}}{D^{\lambda}} \Rightarrow$ Diameters (new gravity flow pipes): $D=\frac{\beta^{1 / \lambda} \cdot Q^{\delta / \lambda}}{J^{1 / \lambda}}$ 
Diameters (new pumping stations): $Q=V e l \cdot \frac{\pi \cdot D^{2}}{4} \Rightarrow D=\left(\frac{4}{\pi \cdot V e l}\right)^{0.5} \cdot Q^{0.5}$

Cost of new pipes:

- gravity flow $C P_{g r N}=K \cdot D^{\alpha} \cdot L=K \cdot \frac{\beta^{(\alpha / \lambda)} \cdot L^{(1+\alpha / \lambda)}}{H_{a v}^{\alpha / \lambda}} \cdot Q^{(\alpha \cdot \delta / \lambda)}$

- pumping stations $C P_{p u N}=K \cdot D^{\alpha} \cdot L=K \cdot\left(\frac{4}{\pi \cdot V e l}\right)^{(0.5 \alpha)} \cdot Q^{(0.5 \alpha)} \cdot L$

Cost of energy (pumping stations):

- new $C E_{p u N}=365 \frac{N H_{\max }}{P_{f}} \cdot \frac{\gamma \cdot Q \cdot\left[\Delta Z+\beta \cdot L \cdot\left(\frac{4}{\pi \cdot V e l}\right)^{(-0.5 \lambda)} \cdot Q^{(\delta-0.5 \lambda)}\right]}{\eta} \cdot C_{k W h} \cdot F$

- existing $C E_{p u E}=365 \frac{N H_{\max , p}}{P_{f}} \cdot \frac{\gamma \cdot Q \cdot\left[\Delta Z+\frac{\beta \cdot L}{D^{\lambda}} \cdot Q^{\delta}\right]}{\eta} \cdot C_{k W h} \cdot F$

Cost of pumps (new pumping stations):

$C P U_{N}=\omega \cdot Q^{\rho} \cdot H_{p u}^{\tau}=\omega \cdot Q^{\rho} \cdot\left[\Delta Z+\beta \cdot L \cdot\left(\frac{4}{\pi \cdot V e l}\right)^{(-0.5 \lambda)} \cdot Q^{(\delta-0.5 \lambda)}\right]^{\tau}$

where: $\quad F=\frac{(1+i n t R)^{n y}-1}{\operatorname{int} R \cdot(1+i n t R)^{n y}}$

and: $Q$ - Flow $\left(\mathrm{m}^{3} / \mathrm{s}\right) ; V-$ Average daily volume $\left(\mathrm{m}^{3}\right) ; P_{f}$ - Peak factor (for example, daily peak factor); $N H_{\max }$ - Maximum number of daily working hours (index $g$ is for gravity flow and $p$ is for pumping stations); $H_{a v}$ - Head available for gravity flow (m); $L$ - length of the pipe (m); $D-$ Diameter of the pipe (m); $C P_{g r N}-$ Cost of pipe for gravity flow (new); $C P_{p u N}-$ Cost of pipe for pumping station (new); $C P U_{N}-$ Cost of pumps for pumping station (new); $C E_{p u N}-$ Cost of energy for pumping station (new); $C E_{p u E}$ - Cost of energy for pumping station (existing); $H_{p u}$ - Pump head (m); $P_{p u}$ - Pump power (kW); $\eta$ - Pump efficiency (-); $\Delta Z$ - Pump geometric head (m); Vel-Reference velocity (for example, $1 \mathrm{~m} / \mathrm{s}) ; \gamma$ - Specific weight of water $\left(\mathrm{kN} / \mathrm{m}^{3}\right) ; N H$ - Average number of daily working hours; $C_{k W h}$ - Cost of energy; int $R$ - Annual interest rate; $n y$ - Number of years of the project plan.

Constraints of physical and planning limitations have been imposed on the model to represent the actual operational characteristics of a given water resources system. With respect to the investigated 
problem, the following considerations are relevant: the total water supplied from each source node $i$ cannot exceed the maximum water supply capacity of the source, the water demand of each user node $j$ has to be fulfilled, the possible flow rate $Q_{i j}$ between already connected nodes $i$ and $j$ cannot exceed the maximum value of $Q_{i j}^{M}$ allowed by the existing pipeline.

\section{Results and discussion}

The optimization model typology is constrained non-linear and was developed in MATLAB using the "fmincon" function, which allows an accurate and expeditious computational evaluation of the optimal solutions. The case study was conducted in the area corresponding to the province of Crotone $(1,717 \mathrm{~km} 2)$ in southern Italy. In this area, 29 springs, 3 intakes and 7 wells are available, for a total drinking water availability of $1,534.8 \mathrm{~L} / \mathrm{s}$ and a total water demand of $922.8 \mathrm{~L} / \mathrm{s}$ (Sogesid, 2001).

Drinking water distribution is carried out within the province of Crotone through six regional water supply systems together with some small municipal systems (Figure 1). The comparison between the water resources distributed by these supply systems and the water needs shows that, although availability exceeds demand, water distribution is not balanced and that, especially in summer, several municipalities are affected by water shortage. This current unbalanced distribution of the available water resources requires an optimization solution.

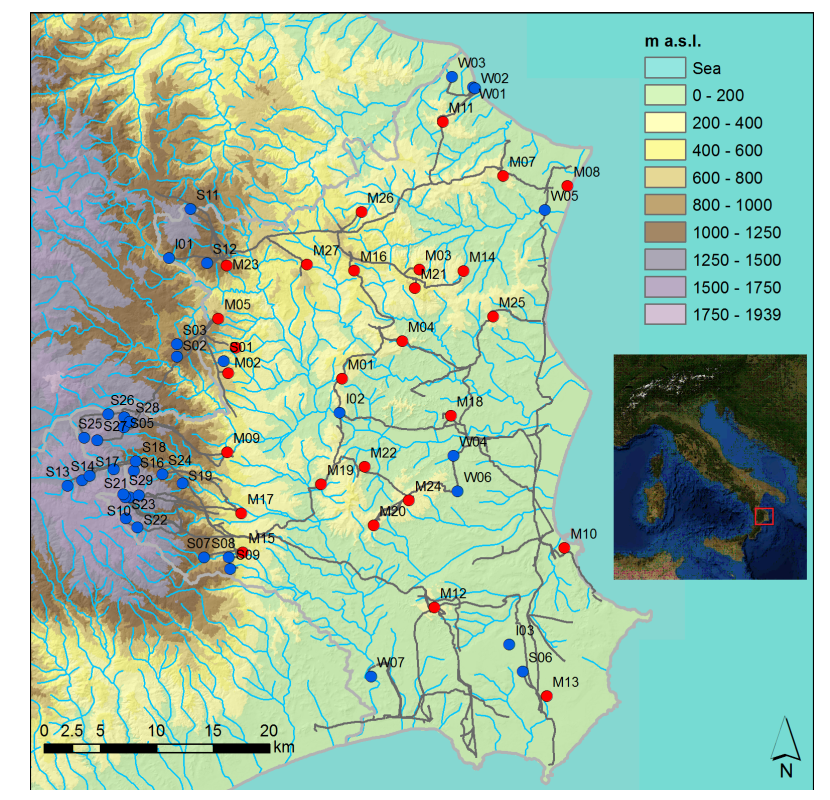

Figure 1: Case study area. Blue circles represent source nodes; red circles represent destination nodes; dark gray lines represent existing conveyance pipes; light gray lines represent administrative borders. 
Results of the future scenario analysis (2016-2050 with RCP4.5) show an average reduction in precipitation of $8.9 \%$ and an average increase in temperature of $1.0{ }^{\circ} \mathrm{C}$ for the whole province of Crotone. An average total runoff reduction for each $5-\mathrm{km}$ resolution cell was estimated of about $19 \%$, while the average contribution to the baseflow decreases on a cell-by-cell basis of about $25 \%$. The main reductions are projected to occur in the central areas of the province where not many source nodes are present. Percentage increase concerns areas with low absolute precipitation values (i.e., the southeast of the province), which cannot contribute significantly to enhance the overall water availability. The reduction in available volumes ranged between $3 \%$ and $54 \%$.
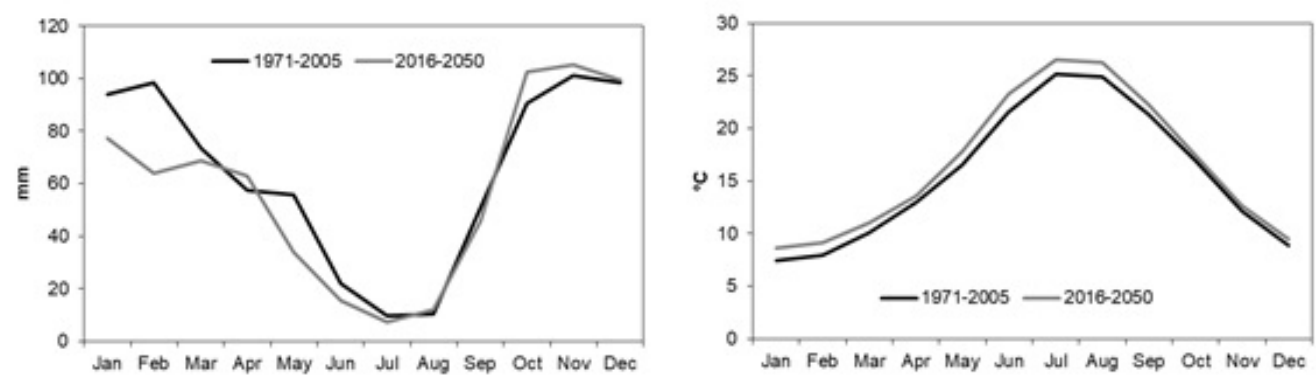

Figure 2: Changes in mean monthly precipitation (\%) and temperature $\left({ }^{\circ} \mathrm{C}\right)(2016-2050$ vs. $1971-2005)$.

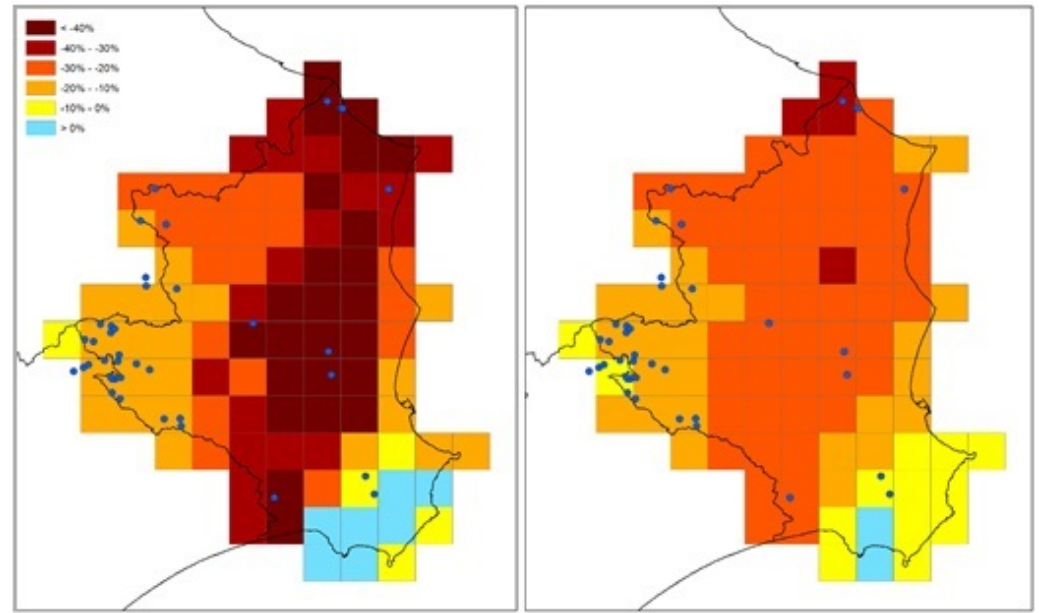

Figure 3: Projected percent changes (2016-2050 vs. 1971-2005) of baseflow (left) and total runoff (right) in the province of Crotone. Source nodes are superimposed.

The optimization model was first applied to the current hydrological scenario. Then, the hydrological model, based on CCLM results, was applied to the future hydrological scenario. This modeling was based on both the current and future available volumes and the various percentage reductions for the different source nodes identified. For the current and future scenarios, the model 
outputs identify suitable solutions for the distribution of water resources in order to satisfy the demand. The outputs of the model identified different solutions for the current scenario and for the climate change scenario, in terms of network sections to be maintained in operation, network sections to be deleted, and new connections to be built. Figure 4 shows a graphic representation of the links between source nodes and destination nodes - at the top, links between source nodes and destination nodes with reference to the current availability; at the bottom, links between source nodes and destination nodes with reference to the future climate change scenario. Black triangles represent source nodes; magenta squares represent destination nodes; blue dotted lines are the existing pipes; blue dashed lines are the parallel pipes; green dashed lines are the parallel pipes present in both scenarios; red lines are the new pipes to be built.
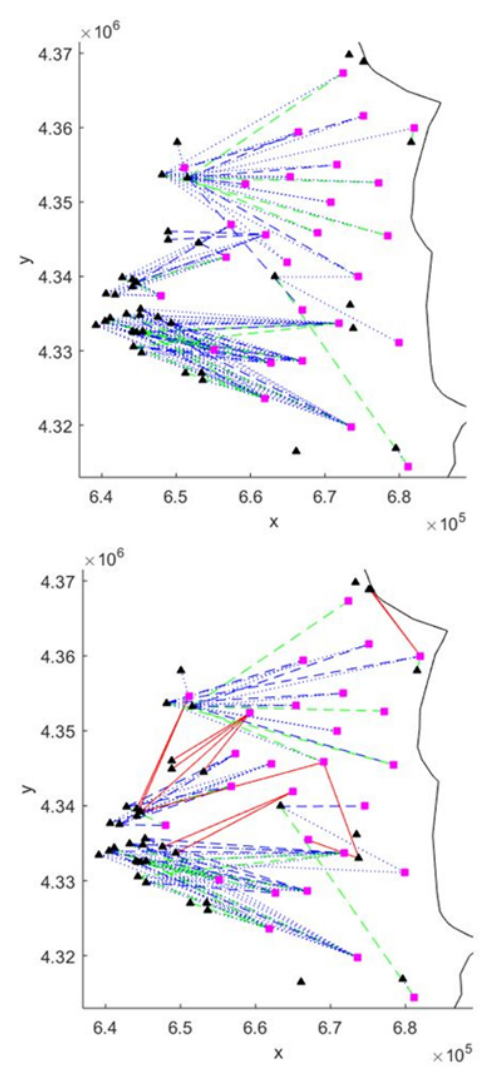

Figure 4: Graphic representation of the links between source nodes and destination nodes (top - current availability, bottom - future climate change scenario). 
Regarding the current scenario, the optimization model includes:

- 122 connections, for a total of $2406 \mathrm{~km}$ of pipes;

- the existing pumping stations are deleted;

- 122 out of the 138 currently existing connections are maintained with 43 pipes in parallel;

- overall, this solution, compared to a topological abstraction of the network, results in a reduction of the overall length of the entire system of about $9 \%$.

For the case of the climate change scenario, the optimization model includes:

- 103 connections for a total of $2067 \mathrm{~km}$ of pipes;

- the existing pumping stations are deleted;

- 90 out of the 138 currently existing connections are maintained with 42 parallel pipes;

- 13 new pipes have to be built;

- overall, this solution, compared to a topological abstraction of the network, results in a reduction of the overall length of the entire system of about $21 \%$.

Regarding parallel pipes, it should be noted that only 20 parallel pipes are present in both scenarios. The different results achieved in the two scenarios show the impact of climate change on optimization results, and confirm the usefulness of planning interventions based on climate change scenario optimization. In fact, if an investment plan only relies on optimization results related to the current water availability, it should be probably integrated in the future, as 23 parallel pipes would no longer be necessary.

This difference shows the importance and the need to assess the impacts of climate change in investment planning. In fact, the investment plan should integrate both scenarios because they lead to different solutions.

\section{Conclusion}

This paper describes a research activity aimed at identifying optimized water resource allocation with reference to water supply systems in order to balance availability and needs. In particular, the paper compares two optimization solutions with reference to current availability and a climate change scenario for the case study of the province of Crotone, a sensitive territory to climate change and characterized by a sufficient supply of drinking water, but by an unbalanced distribution.

The proposed methodology responds to the need for predicting optimized network developments by providing its total length and the length of new connections required, allowing the assessment of the financial impact within an investment plan.

The results of this study are limited only to one future climate projection, therefore they cannot account for uncertainty issues, which are important for long-term planning. Nevertheless, they clearly show the importance of comparing current availability and climate change scenarios in order to better assess the intervention strategies to be adopted. 


\section{References}

Bates, B., Kundzewicz, Z., Wu, S., \& Palutikof, J. (2008). Eds., 2008:Climate Change and Water.Tech. Paper of the Intergovernmental Panel of Climate Change. Geneva: IPCC Secretariat.

Carini , M., Maiolo, M., Pantusa, D., Chiaravalloti, F., \& Capano, G. (2017). Modelling and optimization of least-cost water distribution networks with multiple supply sources and users: A Matlab-based approach. Ricerche di Matematica.

Cervarolo, G., Mendicino , G., \& Senatore, A. (2012). Re-modulating water allocation in a complex multi-reservoir system under current and climate change scenarios. European Water, 47-57.

Jacob , D., Petersen, J., Eggert , B., Alias , A., Christensen, O., Bouwer, L., et al. (2014). EUROCORDEX: new high-resolution climate change projections for European impact research. Regional Environmental Change, 563-578.

Maiolo , M., Mendino , G., Pantusa , D., \& Senatore, A. (2017). Optimization of Drinking Water Distribution Systems in Relation to the Effects of Climate Change. Water, 803.

Maiolo, M., \& Pantusa, D. (2016). An optimization procedure for the sustainable management of water resources. Water Science and Technology: Water Supply, 61-69.

Mendicino, G., Senatore, A., \& Versace, P. (2008 a). A Groundwater Resource Index (GRI) for drought monitoring and forecasting in a mediterranean climate. Journal of Hydrology, 282302.

Mendicino, G., Senatore , A., \& Versace, P. (2008 b). Water resources management in agriculture under drought and water shortage conditions: A case study in southern Italy. European Water, 41-56.

Moss, R. H., Edmonds , J. A., Hibbard, K. A., Manning, M. R., Rose , S. K., Van Vuuren, D., et al. (2010). The next generation of scenarios for climate change research and assessment. Nature, 747-756.

Rockel, B., \& Geyer, B. (2008). The performance of the regional climate model CLM in different Climate regions, based on the example of precipitation. Meteorol.Z., 487-498.

Sogesid. (2001). Accertamento dello stato selle opere, degli impianti di acquedotto e fognature nel Mezzogiorno. Roma: Sogesid S.p.a. 